Published in final edited form as:

Basic Res Cardiol. 2017 July ; 112(4): 48. doi:10.1007/s00395-017-0639-9.

\title{
Exogenous GDF11 Induces Cardiac and Skeletal Muscle Dysfunction and Wasting
}

\author{
Teresa A. Zimmers ${ }^{1,2,3,5,6}$, Yanling Jiang ${ }^{1}$, Meijing Wang ${ }^{1}$, Tiffany W. Liang ${ }^{1}$, Joseph E. \\ Rupert $^{1,3}$, Ernie D. Au ${ }^{1,3}$, Francesco E. Marino ${ }^{1}$, Marion E. Couch ${ }^{4,5,6}$, and Leonidas G. \\ Koniaris $1,5,6$ \\ ${ }^{1}$ Department of Surgery, Indiana University School of Medicine, Indianapolis, IN 46202 USA \\ ${ }^{2}$ Department of Anatomy and Cell Biology, Indiana University School of Medicine, Indianapolis, IN \\ 46202 USA \\ ${ }^{3}$ Department of Biochemistry and Molecular Biology, Indiana University School of Medicine, \\ Indianapolis, IN 46202 USA \\ ${ }^{4}$ Department of Otolaryngology--Head and Neck Surgery, Indiana University School of Medicine, \\ Indianapolis, IN 46202 USA \\ 5IUPUI Center for Cachexia Research, Innovation and Therapy, Indiana University School of \\ Medicine, Indianapolis, IN 46202 USA \\ ${ }^{6}$ IU Simon Cancer Center, Indiana University School of Medicine, Indianapolis, IN 46202 USA
}

\section{SUMMARY}

Growth differentiation factor 11 (GDF11), a TGF-beta super-family member, is highly homologous to myostatin and essential for embryonic patterning and organogenesis. Reports of GDF11 effects on adult tissues are conflicting, with some describing anti-aging and proregenerative activities on the heart and skeletal muscle while others opposite or no effects. Herein, we sought to determine the in vivo cardiac and skeletal muscle effects of excess GDF11. Mice were injected with GDF11 secreting cells, an identical model to that used to initially identify the in vivo effects of myostatin. GDF11 exposure in mice induced whole body wasting and profound loss of function in cardiac and skeletal muscle over a 14-day period. Loss of cardiac mass preceded skeletal muscle loss. Cardiac histologic and echocardiographic evaluation demonstrated loss of ventricular muscle wall thickness, decreased cardiomyocyte size and decreased cardiac function 10 days following initiation of GDF11 exposure. Changes in skeletal muscle after GDF11 exposure were manifest at day 13 and associated with wasting, decreased fiber size, and reduced strength. Changes in cardiomyocytes and skeletal muscle fibers were associated with activation of SMAD2, the ubiquitin-proteasome pathway and autophagy. GDF11 over administration in vivo

Correspondence: Teresa A. Zimmers, PhD, 980 W. Walnut Street, R3-C518, Indianapolis, IN 46202, Phone: 317-278-7289, zimmerst@iu.edu Leonidas G. Koniaris, MD, MBA, FACS, 545 Barnhill Drive, Emerson 511, Indianapolis, IN 46202, Phone: 317-278-7778, 1koniari@iu.edu.

AUTHOR CONTRIBUTIONS

Concept: T.A.Z., M.E.C., L.G.K.; Methodology: T.A.Z., L.G.K.; Formal Analysis: Y.J., T.W.L., M.W., E.D.A., F.E.M., T.A.Z. ; Investigation: Y.J., T.W.L., M.W., E.D.A., F.E.M.; Resources: T.A.Z., L.G.K.; Data Curation: T.W.L., T.A.Z.; Writing-Original Draft: T.W.L.; Writing-Review and Editing: T.A.Z., T.W.L., M.W., E.D.A., M.E.C., L.G.K.; Visualization: Y.J., T.W.L.; Supervision: T.A.Z., L.G.K.; Project Administration: T.A.Z., L.G.K.; Funding Acquisition: T.A.Z., L.G.K. 
results in cardiac and skeletal muscle loss, dysfunction and death. Serum levels of GDF11 by Western blotting were 1.5 fold increased over controls. Although GDF11 effects in vivo are likely dose, route, and duration dependent, its physiologic changes are similar to myostatin and other Activin receptors ligands. These data support that GDF11, like its other closely related TGF-beta family members, induces loss of cardiac and skeletal muscle mass and function.

\section{Keywords}

Growth/differentiation factor-11; GDF11; GDF-11; cachexia; cardiac hypertrophy; SMAD2; atrophy; wasting; autophagy; cardiac function; myostatin; aging

\section{INTRODUCTION}

Growth differentiation factor 11 (GDF11) is a member of the transforming growth factorbeta super-family highly homologous to myostatin/GDF8 [17]. GDF11 was first described as a secreted factor involved in tooth development [17], axial patterning, and kidney organogenesis [15]. More recently, work in adult mice reports regenerative activities for GDF11, with some describing it as the "elixir of youth" because it appeared to decline with age, and exogenous dosing reversed cardiac hypertrophy and improved skeletal muscle in aged animals $[1,12,27]$. Subsequent research questioned these initial conclusions, with disagreements arising regarding the quality and accuracy of the reagents and assays used in the experimental procedures as well as in the interpretation of the results [6, 20, 24].

Additionally, other groups investigating the effects of GDF11 on the hearts of aged mice did not find a regenerative effect [28].

Understanding the in vivo effects of circulating and secreted TGF-beta family members such as GDF11 is difficult. Limitations and differences in observed results may occur due to recombinant protein activity, route of administration, bioavailability and dosing.

Furthermore, the TGF-beta family is highly regulated[23] by a variety of circulating and tissue factors which may alter effects or render it not bioavailable. Presumably all of this variability in delivery may partly explain the varied observations across these different experimental systems. To date, the in vivo effects of secreted proteins including many TGFbeta superfamily members have been identified using injection of $\mathrm{CHO}$ cells selected to express high levels of specific recombinant protein into mice, a classic and powerful approach to evaluating the biological activity of a secreted protein.. This technique leads to sustained, high levels of protein and has been used to identify the cachexia-inducing effects of TNF/cachectin, Interferon- $\gamma$, TGF- $\beta$, IL- 6 and myostatin, all known mediators of muscle wasting $[2,14,19,29,31]$. Furthermore, such an approach has defined hepatotrophic activities of IP-10 and IL-6, anti-apoptotic effects of IL-6, and anti-cachexia activity of follistatin in this fashion $[4,9-11,29,30]$, while others have examined bone formation, tumorigenicity and tumor necrosis, angiogenesis and many other processes in this fashion [7, $22,25,26]$.

Our data presented herein examined the effects of sustained GDF11 on heart and skeletal muscle using CHO cells that were transfected and selected to express high levels of GDF11. We demonstrate that sustained administration of GDF11 in mice injected with GDF11 
expressing cells, but not a variety of control cell lines, produces a systemic wasting syndrome resulting in atrophy of myocardium and skeletal muscle. These data support a strong anti-cardiomyocyte hypertrophic effect for GDF11. It also supports a role for GDF11 in skeletal muscle wasting, an effect suggested by Glass and others [6].

\section{EXPERIMENTAL PROCEDURES}

\section{Mice}

Male athymic nu/nu mice were from Harlan Laboratories, Inc. (Indianapolis, IN, USA). A CHO cell line stably producing GDF11 (CHO-GDF11) was obtained by transfection of the plasmid vector pMSXND containing full-length Gdf11 cDNA into DHFR-deficient CHODUXX cells and subsequent methotrexate selection. A similarly selected $\mathrm{CHO}$ cell line (CHO-control) not expressing detectable recombinant protein was used as a control. Expression of GDF11 was confirmed by Western blotting of conditioned medium, partially purified fractions and serum using anti-GDF11 antibody MAB19581 (R\&D Systems). 10week old male mice were injected in the upper rear portion of the right hind limbs with PBS (normal), CHO-control (Control) or CHO-GDF11 (GDF11) cells in $0.1 \mathrm{~mL}$ of PBS. Mice were euthanized at 10 days (Control and GDF11) or 13 days (normal, Control and GDF11). Studies were done twice; there were no differences between the first and second trials and the second is reported here. Euthanasia was performed by exanguination through cardiac puncture followed by cervical dislocation and tissue collection under isoflurane general anesthesia. Muscles of the left hind limb as well as hearts were collected and weighed, then either snap frozen with liquid nitrogen or embedded in Tissue-Tek® OCT compound (Sakura Finetek USA, Inc., Torrance, CA, USA) and frozen in isopentane. Left tibia length was measured. All experiments were approved by and performed per the guidelines of the Institutional Animal Care and Use Committees at Indiana University School of Medicine.

\section{Echocardiography}

Cardiac muscle dimensions and function were assessed at 8,10, and 13 days after $\mathrm{CHO}$ cell injection via echocardiography using the Vevo® 2100 system (Fujifilm VisualSonics Inc., Toronto, Canada). Mice were placed under isoflurane anesthesia for the duration of the procedure, adjusting the level to maintain a heart rate of $400-500$ beats per minute. Left ventricular dimensions and function were assessed by M-mode scanning of the left ventricular chamber.

\section{Electrocardiography}

Electrocardiogram (ECG) recordings were taken at 7, 9, and 13 days after $\mathrm{CHO}$ cell injection using the ECGenie apparatus (Mouse Specifics, Inc., Boston, MA, USA) as previously described [16]. Awake mice were placed on a platform with footplate electrodes that captures cardiac electrical activity with high fidelity. Several minutes of recording were obtained, and selected $1-5$ second recordings of PQRST complexes were analyzed for various intervals using the eMouse signal analysis software (Mouse Specifics, Inc., Boston, MA, USA). Heart rate and heart rate variability (HRV), calculated as standard deviation of a mean 38 beats, were also obtained. 


\section{Body composition analysis}

Lean and whole body fat masses were measured on awake mice at 9 and 13 days after $\mathrm{CHO}$ cell injection using an EchoMRI-500 (Echo Medical Systems, Houston, TX, USA). Two measurements were taken per mouse per analysis and averaged.

\section{Grip strength measurement}

Grip strength was determined at 10 and 13 days after $\mathrm{CHO}$ cell injection using a force gauge (Extech Intruments, Nashua, NH, USA). Three measurements at each time point were averaged.

\section{Gene expression studies}

qRT-PCR was used to measure the mRNA expression levels of atrogin-1, MuRF1, Pax 7, Mki67, Bnip3, Bnip3L, Myh6, Myh7, Anp, and Bnp in previously snap frozen mouse heart and quadriceps muscle. The total RNA extracted using the Qiagen miRNeasy Mini kit (Valencia, CA, USA) was quantified by NanoDrop (Thermo Scientific, Rockford, IL, USA), and the RNA integrity number was measured with Agilent RNA ScreenTape System (Agilent Technologies, Santa Clara, CA, USA). cDNA was synthesized by Thermo Scientific Verso cDNA synthesis kit. Primers were from Life Technologies. qRT-PCR was conducted on a Roche LightCycler 96 Real-Time PCR System (Roche, Indianapolis, IN, USA) using TaqMan Universal Master Mix II, with UNG (Life Technologies, Grand Island, NY, USA) in a $20 \mu \mathrm{l}$ final reaction mixture. The cycling conditions were as follows: preincubation at $95^{\circ} \mathrm{C}$ for $1 \mathrm{~min}$ and 50 cycles of $10 \mathrm{~s}$ at $95^{\circ} \mathrm{C}$, and $1 \mathrm{~min}$ at $60^{\circ} \mathrm{C}$. Experiments were performed in triplicate for each sample. Results were normalized to either TBP or $18 \mathrm{~S}$, and fold difference was calculated by $2^{-\Delta \Delta \mathrm{Ct}}$.

\section{Western blotting analysis}

Snap frozen mouse heart and quadriceps muscle were homogenized in modified RIPA buffer. Protein was quantified using Pierce BCA protein assay kit (Thermo Scientific, Rockford, IL, USA). Tissue homogenates were separated by polyacrylamide gel (Bio-Rad, Hercules, CA, USA) electrophoresis under reducing conditions and transferred to nitrocellulose membranes (Bio-Rad, Hercules, CA, USA). Immunoblotting used the following antibodies: LC3B (Sigma, Saint Louis, MO, USA); p62 (BD Biosciences, San Jose, CA, USA); pSMAD2, SMAD2, pAKT, AKT, pFOXO3a, FOXO3a, p4E-BP1 70, p4EBP1 65, p4E-BP1 37/46, 4E-BP1, ubiquitin, and GAPDH (Cell Signaling Technology, Danvers, MA, USA). Signal was detected by SuperSignal West Femto (Thermo Scientific, Rockford, IL, USA) or Odyssey CLx western blot detection system (Li-Cor, Lincoln, NE, USA). ImageJ (NIH, Bethesda, MD, USA) or Odyssey software was used for quantification, respectively.

Detection of GDF11 in the circulation was by Western blotting as above of serially diluted serum, running the equivalent of $0.5 \mathrm{ul}$ of serum per lane. GDF11 was detected using antihuman GDF11/BMP11 MAB19581 (R\&D Systems) 


\section{Histology and immunofluorescence}

Snap frozen hearts were cut into $7 \mu \mathrm{m}$ sections and stained with hematoxylin and eosin. Quantification of cardiomyocyte cross-sectional area (CSA) was measured by ImageJ (NIH, Bethesda, MD, USA). Measurements were obtained from ten, non-overlapping 20X fields per mouse. Means were calculated for each animal.

Quadriceps was cut into $10 \mu \mathrm{m}$ section, post-fixed in $4 \%$ paraformaldehyde (Affymetrix; Santa Clara, CA), blocked in $8 \%$ bovine serum albumin and reacted with anti-dystrophin antibody (1:400, Vector Laboratories; Burlingame, CA) overnight at $4^{\circ} \mathrm{C}$. Sections were washed and incubated with a fluorescent secondary antibody (1:1000, Alexa Fluor ${ }^{\circledR} 594$; Fisher Scientific) for one hour at room temperature. Sections were visualized using a fluorescent camera on an Observer.Z1 system (Zeiss; Oberkochen, Germany). Measurements were obtained from ten, non-overlapping 10X fields from each section by ImageJ macro (Minamoto et al., 2007). Mean cross sectional area was calculated for each animal and differences among groups $(\mathrm{N}=4$ normal, $\mathrm{N}=4$ Control, $\mathrm{N}=5$ GDF11) were evaluated by one-way ANOVA.

\section{Statistical analyses}

Unpaired two-sample t-tests for two groups or one-way analysis of variance followed by Tukey's multiple comparisons tests for more than two groups were used as appropriate to evaulate statistical significance with GraphPad Prism version 7, GraphPad Software, La Jolla California USA, www.graphpad.com. For cardiac parameters in Figure 2, results were tested for time dependence within groups by one-way ANOVA followed by test for linear trend and for the entire cohort with two-way ANOVA.

\section{RESULTS}

\section{GDF11 Reduced Overall Body Weight and Organ Size}

Athymic nude mice were divided into three groups - a normal group, a control group in which $\mathrm{CHO}$ cells expressing no recombinant protein were injected, and an experimental group into which $\mathrm{CHO}$ cells expressing murine GDF11 were injected into the right hind limb (Figure S1). Tumors were visible by day 10 after injection and no differences in tumor mass were observed between groups $(0.205 \mathrm{~g} \pm 0.104 \mathrm{GDF} 11$ vs. $0.206 \mathrm{~g} \pm 0.089$ control, $N S$ ). By day 9, body weights of GDF11 mice were significantly reduced compared to the control mice; this difference was even greater at day 13 (Figure 1, S1). Decreased whole body lean mass was not detected until day 13 . There was no difference in percentage whole body fat mass measured between the groups. However, large differences in gross organ mass, including heart and skeletal muscle, were observed at necropsy (Figure S1-3). The differences in body and organ mass were due to weight loss, not suppression of growth because the control group did not grow over time and tibia length was not different among groups at euthanasia (Figure S1). Weights of kidney and liver were reduced by day 10, while weights of the carcass, epididymal fat pad, kidney and liver were reduced by day 13. Lungs and spleen were spared (Figure S2). Overall, circulating levels of GDF11 were increased approximately $37 \%$ overall compared with controls, as assessed by Western blotting of plasma (Figure S1). 


\section{GDF11 Reduced Heart Weight and Cardiomyocyte Size}

There are conflicting reports on the effects of GDF11 administration on mouse heart and cardiomyocyte size [12, 20, 28]. Here, GDF11 decreased heart mass by day 10. Heart mass decreased $27 \%$ overall by day 13 (Figure 1A, B). Heart mass was decreased proportionately to total body mass (Figure 1C). Whole hearts and sections revealed visibly smaller organ and cardiomyocyte size in the GDF11 group (Figure 1D). Mean cardiomyocyte cross-sectional area was reduced approximately 35\%, consistent with the Loffredo and Poggioli studies (Figure 1D-F).

\section{GDF11 Altered Cardiac Proteolytic and Remodeling Pathways}

Expression of genes that normally compensate cardiac dysfunction was generally unchanged with GDF11. Anp and Bnp are typically increased in cardiac dysfunction and exert protective activities in cardiovascular homeostasis [18]. Activation of fetal gene expression, including Myh6 and Myh7 is also often observed in models of heart failure [5]. However, we did not find any changes in Anp, Myh6 (aMHC), or Myh7 ( $\beta \mathrm{MHC}$ ), similar to a recent study of GDF11 in aged mouse hearts [28]. While expression of Bnp at day 10 was increased in the GDF11 group, expression at day 13 was not significantly different (Figure $1 G)$.

GDF11 induced expression of ubiquitin-protein ligases atrogin-1/MAFbx/Fbxo32 and MuRF1/Trim63 at day 10 and MuRF1/Trim63 at day 13 [3, 8] (Figure 1G). Moreover, there was evidence of diminished cellular proliferation with decreased expression of $M k i 67$ and potentially increased apoptosis and autophagy given increased expression of the proapoptotic and mitophagy-associated gene Bnip3 in the GDF11 mice at day 13. Differences in Bnip3L expression were not statistically significant following GDF11 exposure (Figure $1 \mathrm{G})$. This expression profile suggests activation of proteolysis, apoptosis and autophagy by GDF11 in hearts.

Western blotting showed that GDF11 increased cardiac SMAD2 protein on day 13. Phosphorylated SMAD2 also appeared somewhat increased but this did not reach statistical significance (Figure 1H). Total protein ubiquitylation was increased 42\% in GDF11 hearts compared to the control group (Figure S4). There were no statistically significant differences detected in levels of atrophy markers pFOXO3a, FOXO3a, p4E-BP1, or p62 (Figure S4).

\section{GDF11 Reduced Cardiac Size and Function}

Echocardiography supported our findings that GDF11 reduced heart size and also demonstrated reduced function. Cardiac size and dimensions were generally decreased in the GDF11 mice, where significant decreases in left ventricular internal diameter during diastole (LVIDd), posterior wall thickness during systole (PWTs), and left ventricular mass (LVM) were apparent by day 10, then later in posterior wall thickness during diastole (PWTd) by day 13 (Figures 2A-C, S5). GDF11 also significantly decreased stroke volume (SV), ejection fraction (EF), and fractional shortening (FS) by day 10 (Figure 2D-F). Increasing time of GDF11 exposure also correlated with increasing declines in both size (LVIDd, PWTd, PWTs, LVM) and functional measurements (SV, EF, FS) (Figure S6). No changes over time were noted in the control mice and measurements of normal and control mice 
were similar (Figures S6, S7). Electrocardiography was performed to measure heart rate and conductivity; however no differences were observed (Figure S8).

\section{GDF11 Decreased Skeletal Muscle Mass and Strength}

Muscle mass loss followed heart wasting, with individual muscles consistently decreased only on day 13 (Figures 3A-C). Grip strength in the GDF11 mice was also significantly decreased at day 13 (Figure 3D). Mean quadriceps myofiber cross-sectional area (CSA) of the GDF11 group was significantly smaller than controls at day 13 (Figure 3E-G).

\section{GDF11 Induced Atrophy-Associated Pathways in Skeletal Muscle without Effects on Pax7}

GDF11 dramatically induced atrogin-1 and $M u R F 1$ by day 13. Bnip3 expression tended to be increased in the GDF11 group, although Bnip3L was unchanged. Pax 7 was similar between GDF11 and control mice, while Mki67 expression was significantly decreased. Thus GDF11-induced atrophy was apparently not accompanied by cell proliferation or compensatory regeneration (Figure $3 \mathrm{H}$ ).

Western blotting of quadriceps showed increased pSMAD2 with GDF11, while total SMAD2 was unchanged between GDF11 mice and controls and decreased compared to normal mice (Figure 4A). Phosphorylated and total AKT were unchanged, as were pFOXO3a and FOXO3a (Figure 4B), and FOXO1 was not detected. Expression of the translation repressor 4E-BP1 was increased while its phosphorylated (inactive) forms were decreased by GDF11, suggesting that translation was inhibited (Figure 4C). Consistent with the trend towards increased Bnip3, levels of the autophagy proteins LC3-II, LC3-II/LC3-I, and p62 (Figure 4D) were increased by GDF11. Total protein ubiquitylation was also markedly increased (Figure 4E), consistent with the up-regulation of ubiquitin ligases atrogin-1/MAFbx and MuRF1 mRNAs. No increase in proteasome activity was detected at this time point, however.

\section{DISCUSSION}

Our results show that excess GDF11 in adult mice leads to cachexia, with consequential cardiac and skeletal muscle dysfunction and weakness. These results are consistent with shared structure and function to myostatin, which is $90 \%$ homologous, exerts similar wasting effects [29] and binds to the same receptor complex of type I (ALK5, ALK6) and type II (ACVR2A, ACVR2B) receptors. Activin A and Activin B in excess also produce a similar cachexia phenotype. Of these, thus far only Activin has been convincingly linked to a human cachexia condition, i.e. weight loss in cancer. However GDF11 has not been as carefully studied in diseases associated with cardiac and muscle loss due to limitations in available assays.

Initial studies $[12,27]$ showed that GDF11 declines with age, and that the reintroduction of this "elixir of youth" [1] reverses age-related degenerative changes in the body, including cardiac hypertrophy and skeletal muscle dysfunction/atrophy [12, 20, 27]. However in vivo studies by Smith et al. reported no changes in cardiac structure or function with GDF11 administration [28]. Thus, we believe that the previous finding of cardiac hypertrophy reversal with GDF11 might not represent reversion to a normal cardiac phenotype, but rather 
induction of cardiac atrophy. Similarly, we also find that GDF11 overexpression leads to skeletal muscle wasting.

There are conflicting research findings on body composition changes after GDF11 administration. Poggioli et al. briefly mentioned weight loss in both young and old mice given $0.5 \mathrm{mg} / \mathrm{kg} /$ day and $/$ or $1 \mathrm{mg} / \mathrm{kg} /$ day of exogenous GDF11 but this finding was not explored in more detail [20]. Sinha et al. used $0.1 \mathrm{mg} / \mathrm{kg} / \mathrm{day}$ and showed that body weight and muscle mass (tibialis and extensor digitorum longus) were unchanged in both young and old mice [27]. We find that GDF11 caused body weight loss, largely due to loss in lean mass. Skeletal muscle mass also significantly decreased with GDF11 overexpression. The differences between our study and Sinha et al. could potentially be attributed to differences in circulating GDF11 dose.

There have also been conflicting findings on changes in the heart with GDF11 administration. Loffredo et al. showed that intraperitoneal GDF11 administration resulted in decreased heart weight and decreased cardiomyocyte cross-sectional area, but no difference in echocardiography compared to controls [12]. Similarly, Poggioli et al showed that for both young and old mice, intraperitoneal GDF11 administration decreased heart weight as well as cross-sectional area of cardiomyocytes [20]. However, in the Smith et al. study, i.p. GDF11 administration in 24 month old mice resulted in no differences in heart mass, echocardiography, terminal hemodynamic studies, cardiomyocyte cross-sectional area, or amount of fibrosis compared to controls [28]. Our study supports that GDF11 promotes cardiac atrophy with corresponding decreases in both heart size and function.

The mechanisms by which GDF11 activated cardiac wasting are unclear. Our results suggest enhanced autophagy and apoptosis, without altering expression of the genes that are typically induced in heart failure. In one study of failing hearts (presumably with a hypertrophic component), the investigators found that ANP levels are greater while aMHC and $\beta \mathrm{MHC}$ levels are decreased compared to non-failing hearts [21]. In another study, however, cardiac hypertrophy due to pressure overload was found to increase both aMHC and $\beta$ MHC levels in cardiomyocytes [13]. While these studies differed in the direction of change in the cardiac markers, they nevertheless demonstrated that the levels of cardiac markers are changed by hypertrophic cardiac pathology. In accordance with Smith et al., we also found no differences in levels of cardiac markers (ANP, BNP, aMHC, and $\beta M H C$ ) that typically accompany pathologic hypertrophy [28],. These findings suggest that the atrophic effects of GDF11 likely do not act by diminishing pathologic cardiac hypertrophy.

Regarding skeletal muscle regenerative potential, Sinha and Jang et al showed that GDF11 supplementation in old mice increased the frequency and function of muscle satellite cells as well as improved exercise endurance and grip strength [27]. Conversely, Egerman et al showed that GDF11 treatment did not affect regenerative capacity or cross-sectional area of skeletal muscle after injury; in fact, in vitro studies showed that GDF11 decreased the growth of adult and aged muscle satellite cells and did not change the expression of markers of muscle differentiation [6]. Our study observed that GDF11 activated muscle atrophy pathways, including autophagy and ubiquitylation, while inducing inhibitors of translation. This led to $20-30 \%$ decreased skeletal muscle mass and a corresponding $20 \%$ decrease in 
myofiber cross sectional area. Consistent with reduced muscle mass, the grip strength measurements also declined. Unchanged Pax7 levels and decreased Ki67 expression suggests that GDF11 in this setting failed to activate regeneration. Muscle loss occurred later than heart wasting, suggesting that there might be differences in sensitivity to GDF11 in cardiac versus skeletal muscle, with heart wasting induced at lower doses or shorter duration. Alternatively, wasting of skeletal muscle might have been initiated secondary to reductions in heart function.

While this study contributes important evidence around GDF11 function, it has limitations. Our study used only 10-week old (young) mice, thus does not address aging. Although reliability of various quantification methods for GDF11 has been brought into question, Western blotting of plasma using an antibody validated to detect GDF11 showed that our intervention increased circulating GDF11 levels by approximately $40 \%[6,20,28]$, with several samples overlapping the range of normal. We cannot exclude the possibility of underlying, compensatory dysregulation of endogenous GDF11 expression or changes in expression of critical components of GDF11 regulation, including activating proteases, inhibitory binding proteins, receptors and signaling modulators. However, no prior studies have examined these in detail either, which is a general limitation of this field. Given its complex post-transcriptional regulation, indeed it is naïve to focus solely on mRNA and protein levels of GDF11 (REF).

Overall, our data demonstrate that GDF11 administration to adult mice causes significant weight loss and cachexia, as well as cardiac and skeletal muscle dysfunction and weakness. Thus any endeavors to use GDF11 as a therapeutic agent should be approached with caution.

\section{Supplementary Material}

Refer to Web version on PubMed Central for supplementary material.

\section{Acknowledgments}

Supported by National Cancer Institute grants R01 CA194593, R01 CA122596, National Institute for General Medical Studies grant R01 GM092758, and Pennsylvania Department of Health CURE Grant TJU\# 080-37038AI0801 to T.A.Z., and by the Lilly Endowment, Inc. Physician Scientist Initiative to L.G.K. The authors used resources of the IU Laboratory Animal Resource Center and the IU school of Medicine Small Animal Ultrasound Core.

\section{References}

1. Andersen RE, Lim DA. An ingredient for the elixir of youth. Cell Res. 2014; 24:1381-1382. DOI: 10.1038/cr.2014.107 [PubMed: 25112712]

2. Black K, Garrett IR, Mundy GR. Chinese hamster ovarian cells transfected with the murine interleukin-6 gene cause hypercalcemia as well as cachexia, leukocytosis and thrombocytosis in tumor-bearing nude mice. Endocrinology. 1991:2657-2659. [PubMed: 2019273]

3. Bodine SC, Latres E, Baumhueter S, Lai VK, Nunez L, Clarke BA, Poueymirou WT, Panaro FJ, Na E, Dharmarajan K, Pan ZQ, Valenzuela DM, DeChiara TM, Stitt TN, Yancopoulos GD, Glass DJ. Identification of ubiquitin ligases required for skeletal muscle atrophy. Science (New York, NY). 2001; 294:1704-1708. DOI: 10.1126/science.1065874

4. Bonetto A, Aydogdu T, Jin X, Zhang Z, Zhan R, Puzis L, Koniaris LG, Zimmers TA. JAK/STAT3 pathway inhibition blocks skeletal muscle wasting downstream of IL-6 and in experimental cancer 
cachexia. Am J Physiol Endocrinol Metab. 2012; 303:E410-421. DOI: 10.1152/ajpendo. 00039.2012 [PubMed: 22669242]

5. Dirkx E, da Costa Martins PA, De Windt LJ. Regulation of fetal gene expression in heart failure. Biochim Biophys Acta. 2013; 1832:2414-2424. DOI: 10.1016/j.bbadis.2013.07.023 [PubMed: 24036209]

6. Egerman MA, Cadena SM, Gilbert JA, Meyer A, Nelson HN, Swalley SE, Mallozzi C, Jacobi C, Jennings LL, Clay I, Laurent G, Ma S, Brachat S, Lach-Trifilieff E, Shavlakadze T, Trendelenburg AU, Brack AS, Glass DJ. GDF11 Increases with Age and Inhibits Skeletal Muscle Regeneration. Cell Metab. 2015; 22:164-174. DOI: 10.1016/j.cmet.2015.05.010 [PubMed: 26001423]

7. Gitelman SE, Kobrin MS, Ye JQ, Lopez AR, Lee A, Derynck R. Recombinant Vgr-1/BMP-6expressing tumors induce fibrosis and endochondral bone formation in vivo. J Cell Biol. 1994; 126:1595-1609. [PubMed: 8089189]

8. Gomes MD, Lecker SH, Jagoe RT, Navon A, Goldberg AL. Atrogin-1, a muscle-specific F-box protein highly expressed during muscle atrophy. Proc Natl Acad Sci U S A. 2001; 98:14440-14445. DOI: 10.1073/pnas.251541198 [PubMed: 11717410]

9. Jin X, Zhang Z, Beer-Stolz D, Zimmers TA, Koniaris LG. Interleukin-6 inhibits oxidative injury and necrosis after extreme liver resection. Hepatology (Baltimore, Md). 2007; 46:802-812. DOI: 10.1002/hep. 21728

10. Jin X, Zimmers TA, Perez EA, Pierce RH, Zhang Z, Koniaris LG. Paradoxical effects of short- and long-term interleukin-6 exposure on liver injury and repair. Hepatology (Baltimore, Md). 2006; 43:474-484. DOI: 10.1002/hep.21087

11. Koniaris LG, Zimmers-Koniaris T, Hsiao EC, Chavin K, Sitzmann JV, Farber JM. Cytokineresponsive gene-2/IFN-inducible protein-10 expression in multiple models of liver and bile duct injury suggests a role in tissue regeneration. J Immunol. 2001; 167:399-406. [PubMed: 11418676]

12. Loffredo FS, Steinhauser ML, Jay SM, Gannon J, Pancoast JR, Yalamanchi P, Sinha M, Dall'Osso C, Khong D, Shadrach JL, Miller CM, Singer BS, Stewart A, Psychogios N, Gerszten RE, Hartigan AJ, Kim MJ, Serwold T, Wagers AJ, Lee RT. Growth differentiation factor 11 is a circulating factor that reverses age-related cardiac hypertrophy. Cell. 2013; 153:828-839. DOI: 10.1016/j.cell.2013.04.015 [PubMed: 23663781]

13. Lopez JE, Myagmar BE, Swigart PM, Montgomery MD, Haynam S, Bigos M, Rodrigo MC, Simpson PC. beta-myosin heavy chain is induced by pressure overload in a minor subpopulation of smaller mouse cardiac myocytes. Circ Res. 2011; 109:629-638. DOI: 10.1161/CIRCRESAHA. 111.243410 [PubMed: 21778428]

14. Matthys P, Dijkmans R, Proost P, Van Damme J, Heremans H, Sobis H, Billiau A. Severe cachexia in mice inoculated with interferon-gamma-producing tumor cells. Int J Cancer. 1991; 49:77-82. [PubMed: 1908442]

15. McPherron AC, Lawler AM, Lee SJ. Regulation of anterior/posterior patterning of the axial skeleton by growth/differentiation factor 11. Nat Genet. 1999; 22:260-264. [PubMed: 10391213]

16. Mielcarek M, Inuabasi L, Bondulich MK, Muller T, Osborne GF, Franklin SA, Smith DL, Neueder A, Rosinski J, Rattray I, Protti A, Bates GP. Dysfunction of the CNS-heart axis in mouse models of Huntington's disease. PLoS Genet. 2014; 10:e1004550.doi: 10.1371/journal.pgen.1004550 [PubMed: 25101683]

17. Nakashima M, Toyono T, Akamine A, Joyner A. Expression of growth/differentiation factor 11, a new member of the BMP/TGFbeta superfamily during mouse embryogenesis. Mechanisms of development. 1999; 80:185-189. [PubMed: 10072786]

18. Nishikimi T, Maeda N, Matsuoka H. The role of natriuretic peptides in cardioprotection. Cardiovasc Res. 2006; 69:318-328. DOI: 10.1016/j.cardiores.2005.10.001 [PubMed: 16289003]

19. Oliff A, Defeo-Jones D, Boyer M, Martinez D, Kiefer D, Vuocolo G, Wolfe A, Socher SH. Tumors secreting human TNF/cachectin induce cachexia in mice. Cell. 1987; 50:555-563. [PubMed: 3607879]

20. Poggioli T, Vujic A, Yang P, Macias-Trevino C, Uygur A, Loffredo FS, Pancoast JR, Cho M, Goldstein J, Tandias RM, Gonzalez E, Walker RG, Thompson TB, Wagers AJ, Fong YW, Lee RT. Circulating Growth Differentiation Factor 11/8 Levels Decline With Age. Circ Res. 2016; 118:29_ 37. DOI: 10.1161/CIRCRESAHA.115.307521 [PubMed: 26489925] 
21. Razeghi P, Young ME, Alcorn JL, Moravec CS, Frazier OH, Taegtmeyer H. Metabolic gene expression in fetal and failing human heart. Circulation. 2001; 104:2923-2931. [PubMed: 11739307]

22. Richter G, Kruger-Krasagakes S, Hein G, Huls C, Schmitt E, Diamantstein T, Blankenstein T. Interleukin 10 transfected into Chinese hamster ovary cells prevents tumor growth and macrophage infiltration. Cancer Res. 1993; 53:4134-4137. [PubMed: 8364905]

23. Robertson IB, Rifkin DB. Regulation of the Bioavailability of TGF-beta and TGF-beta-Related Proteins. Cold Spring Harb Perspect Biol. 2016; :8.doi: 10.1101/cshperspect.a021907

24. Rodgers BD, Eldridge JA. Reduced Circulating GDF11 Is Unlikely Responsible for AgeDependent Changes in Mouse Heart, Muscle, and Brain. Endocrinology. 2015; 156:3885-3888. DOI: 10.1210/en.2015-1628 [PubMed: 26372181]

25. Sgadari C, Angiolillo AL, Cherney BW, Pike SE, Farber JM, Koniaris LG, Vanguri P, Burd PR, Sheikh N, Gupta G, Teruya-Feldstein J, Tosato G. Interferon-inducible protein-10 identified as a mediator of tumor necrosis in vivo. Proc Natl Acad Sci U S A. 1996; 93:13791-13796. [PubMed: 8943014]

26. Shimizu K, Yoshikawa H, Matsui M, Masuhara K, Takaoka K. Periosteal and intratumorous bone formation in athymic nude mice by Chinese hamster ovary tumors expressing murine bone morphogenetic protein-4. Clin Orthop Relat Res. 1994:274-280.

27. Sinha M, Jang YC, Oh J, Khong D, Wu EY, Manohar R, Miller C, Regalado SG, Loffredo FS, Pancoast JR, Hirshman MF, Lebowitz J, Shadrach JL, Cerletti M, Kim MJ, Serwold T, Goodyear LJ, Rosner B, Lee RT, Wagers AJ. Restoring systemic GDF11 levels reverses age-related dysfunction in mouse skeletal muscle. Science (New York, NY). 2014; 344:649-652. DOI: $10.1126 /$ science. 1251152

28. Smith SC, Zhang X, Zhang X, Gross P, Starosta T, Mohsin S, Franti M, Gupta P, Hayes D, Myzithras M, Kahn J, Tanner J, Weldon SM, Khalil A, Guo X, Sabri A, Chen X, MacDonnell S, Houser SR. GDF11 Does Not Rescue Aging-Related Pathological Hypertrophy. Circ Res. 2015; doi: 10.1161/circresaha.115.307527

29. Zimmers TA, Davies MV, Koniaris LG, Haynes P, Esquela AF, Tomkinson KN, McPherron AC, Wolfman NM, Lee SJ. Induction of cachexia in mice by systemically administered myostatin. Science (New York, NY). 2002; 296:1486-1488. [pii]. DOI: 10.1126/science. 1069525296/5572/1486

30. Zimmers TA, McKillop IH, Pierce RH, Yoo JY, Koniaris LG. Massive liver growth in mice induced by systemic interleukin 6 administration. Hepatology (Baltimore, Md). 2003; 38:326-334.

31. Zugmaier G, Paik S, Wilding G, Knabbe C, Bano M, Lupu R, Deschauer B, Simpson S, Dickson RB, Lippman M. Transforming growth factor beta 1 induces cachexia and systemic fibrosis without an antitumor effect in nude mice. Cancer Res. 1991:3590-3594. [PubMed: 2054795] 

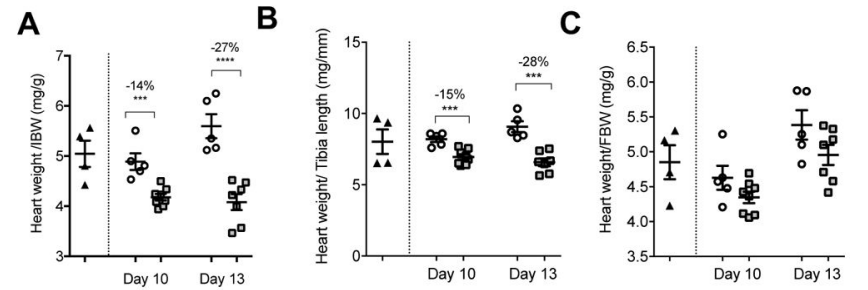

D

\ Normal • CONT a GDF11
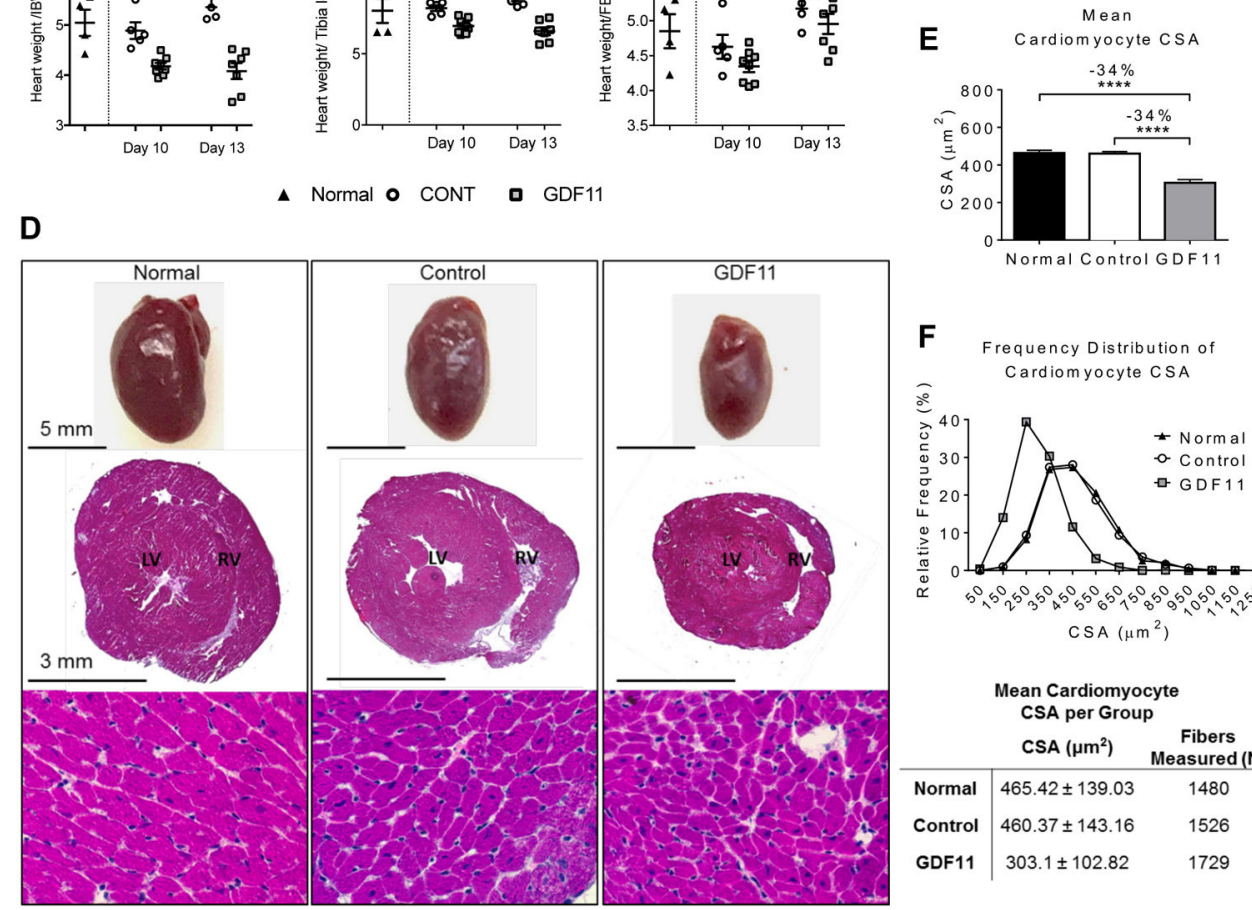

G
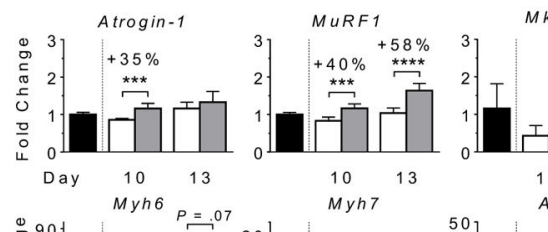

Mki67
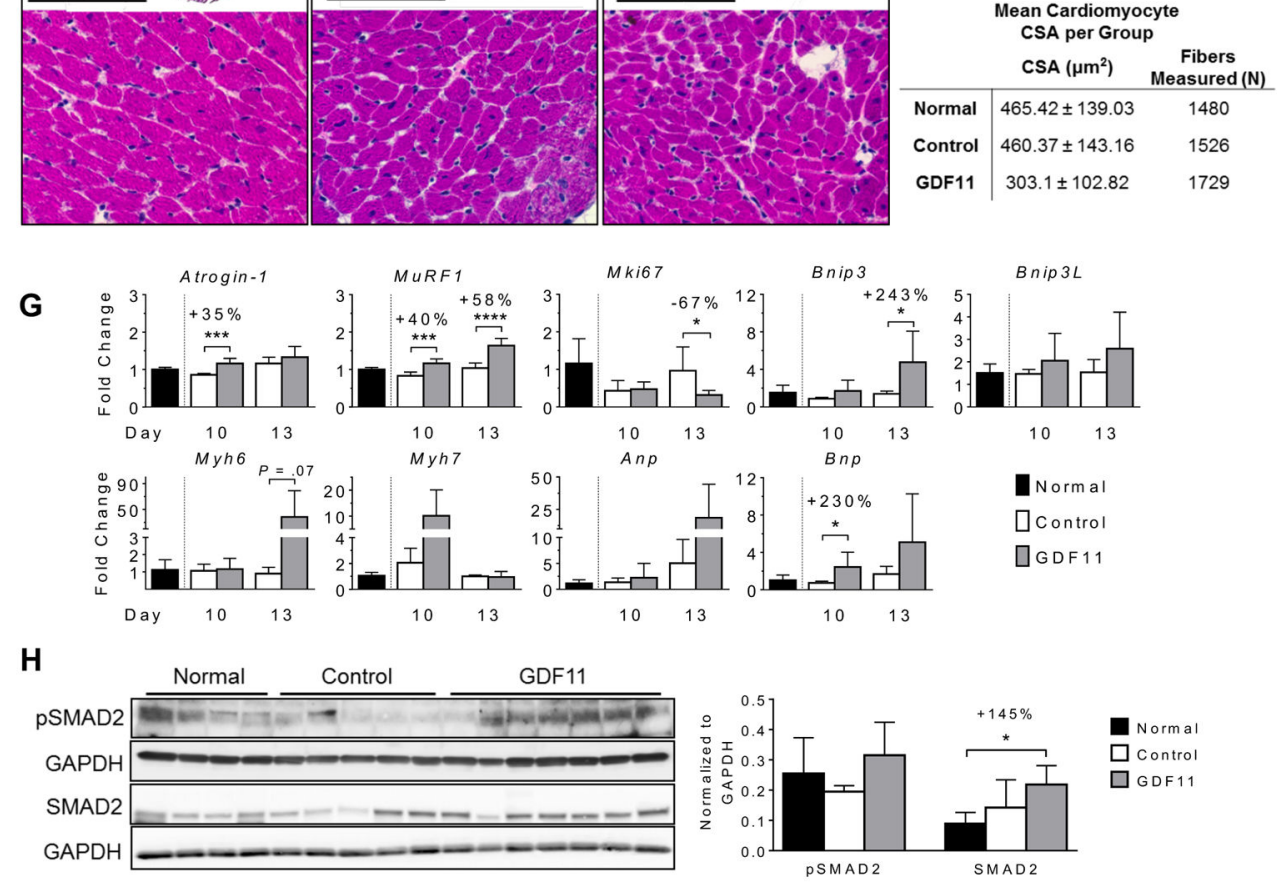

Figure 1. Heart and Cardiomyocyte Size Decrease with GDF11 Overexpression (A and B) Heart weights (HW) of normal, control, and GDF11 mice at sacrifice, normalized to (A) initial body weight (IBW), (B) tibia length (TL), and (C) to final body weight (FBW). Heart weights of normal mice $(n=4)$ taken at day 13 are shown to the left of the dashed vertical line, and those of control and GDF11 mice at day 10 ( $n=5$ and $n=8$, respectively) and at day 13 ( $n=5$ and $n=7$, respectively) are compared to the right of the line. Horizontal lines with error bars indicate mean \pm SEM. 
(D) Gross anatomy (top row of figure on left, $5 \mathrm{~mm}$ scale bar) and hematoxylin and eosin staining (cross-section, middle row, $3 \mathrm{~mm}$ scale bar; 40x magnification, bottom row) of normal, control, and GDF11 mouse hearts at day 13. LV, left ventricle; RV, right ventricle. (E) Mean cardiomyocyte fiber cross-sectional area (CSA) of normal, control, and GDF11 mice at day 13. Data are mean \pm SD.

(F) Histogram of cardiomyocyte CSA for normal, control, and GDF11 mice at day 13. Numerical data of mean cardiomyocyte CSA (data are mean \pm SEM) and number of fibers measured are shown in table below.

(G) Gene expression analyses of cardiac muscle in normal (left of dashed vertical line, measured at day 13), control, and GDF11 mice at days 10 and 13. Data are represented as mean fold change $\pm \mathrm{SD}$. Fold change for atrogin- 1 and $M u R F 1$ were calculated using reference gene TBP, while data for all other genes were calculated using reference gene $18 \mathrm{~S}$. (H) Western blotting analyses to determine SMAD2 levels in normal, control, and GDF11 mice at day 13. Horizontal lines with error bars indicate mean \pm SD.

Statistically significant differences are indicated at $\mathrm{p}<0.05(*), \mathrm{p}<0.01(* *), \mathrm{p}<0.001$ $(* * *)$ and $\mathrm{p}<0.0001$ (****). 

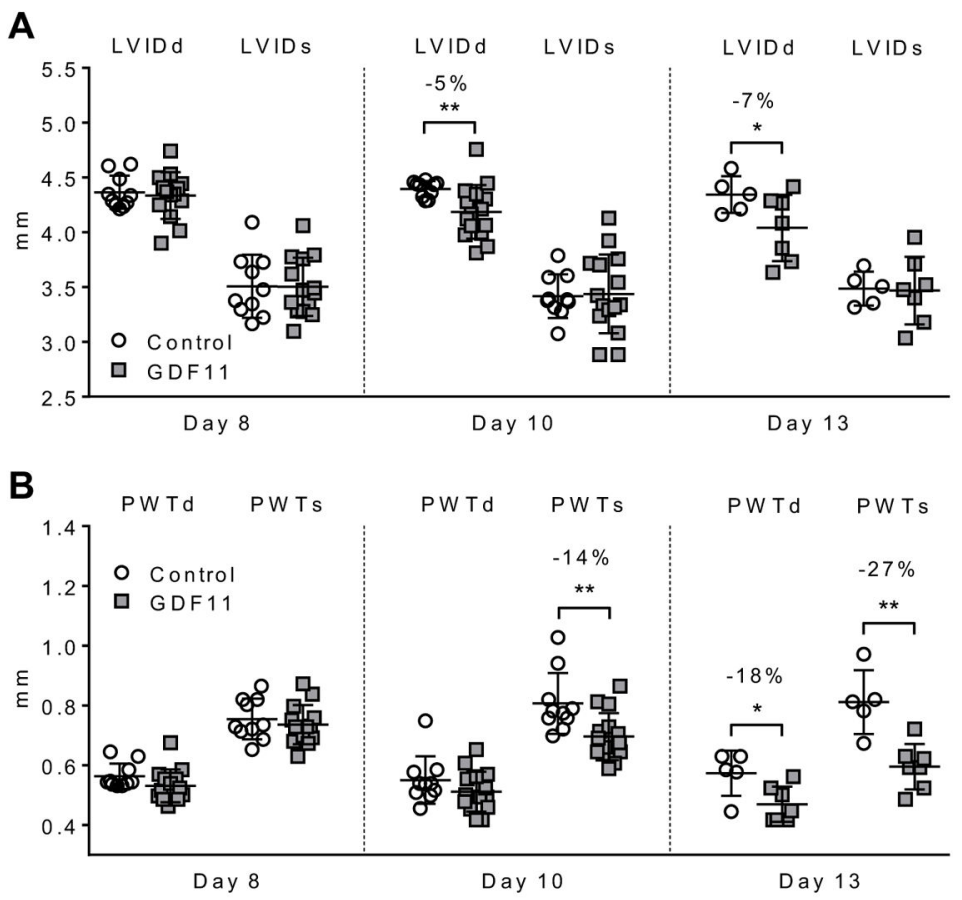

C
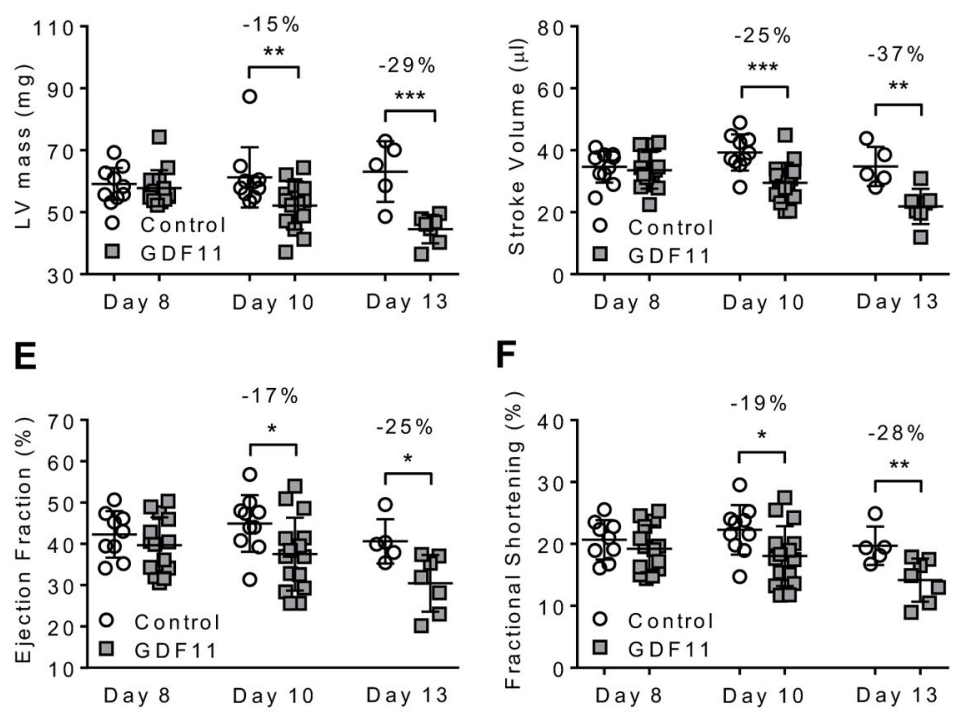

Figure 2. Echocardiographic Measurements in Mice with GDF11 Overexpression Demonstrate Reduced Cardiac Size and Function

(A and B) Time course of echocardiographic dimension changes in control and GDF11 mice. (A) Left ventricular internal diameter (diastole/systole) (LVIDd/s) and (B) posterior wall thickness (diastole/systole) (PWTd/s) measurements are shown.

$(\mathrm{C}-\mathrm{F})$ Time course of changes in echocardiographic measurements of (C) left ventricle (LV) mass and cardiac function as measured by (D) stroke volume, (E) ejection fraction, and (F) fractional shortening. Horizontal lines with error bars indicate mean \pm SD.

Statistically significant differences are indicated at $\mathrm{p}<0.05(*), \mathrm{p}<0.01(* *)$, and $\mathrm{p}<0.001$ $(* * *)$. 

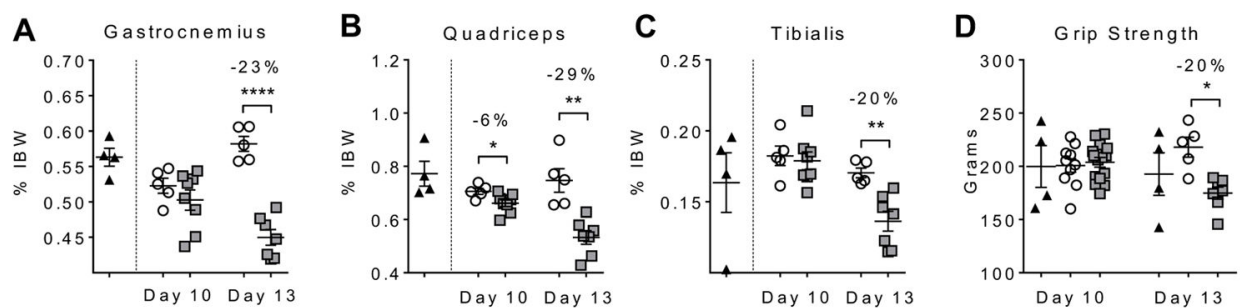

E

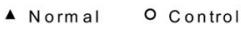

口 GDF11

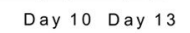

$\mathbf{F}$

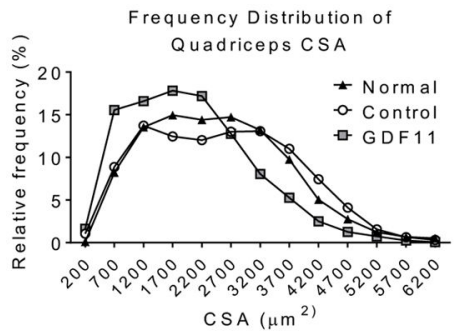

G

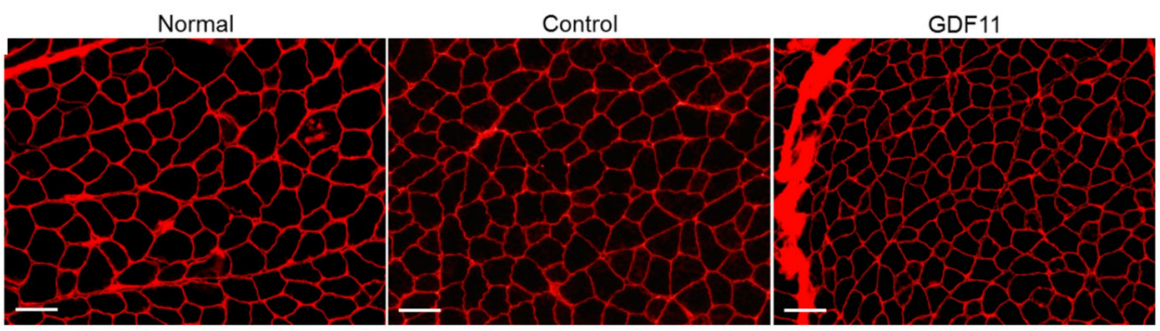

Mean Myocyte CSA per Group

\begin{tabular}{|c|c|c|}
\hline & $\operatorname{csA}\left(\mu \mathrm{m}^{2}\right)$ & $\begin{array}{c}\text { Fibers } \\
\text { Measured (N) }\end{array}$ \\
\hline Normal & $2521 \pm 87.5$ & 4618 \\
\hline Control & $2565 \pm 85.2$ & 4690 \\
\hline GDF11 & $2035 \pm 67.8$ & 5387 \\
\hline
\end{tabular}

H
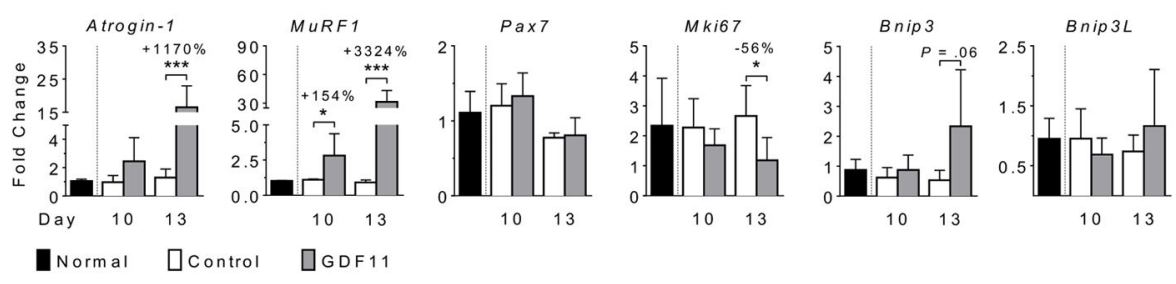

Figure 3. Skeletal Muscle Weight and Function Decrease with GDF11 Overexpression (A-C) Weights of the left hind limb (A) gastrocnemius, (B) quadriceps, and (C) tibialis muscles taken at sacrifice were reported as percentages of initial body weight (IBW). Muscle weights of normal mice $(n=4)$ taken at day 13 are shown to the left of the dashed vertical line, and those of control and GDF11 mice at day 10 ( $n=5$ and $n=8$, respectively) and at day 13 ( $n=5$ and $n=7$, respectively) are compared to the right of the line. Horizontal lines with error bars indicate mean \pm SEM.

(D) Grip strength measurements at day 10 and 13. Each data point is an average of 3 separate measurements in each mouse. Horizontal lines with error bars indicate mean \pm SEM. 
(E) Mean quadriceps myocyte fiber cross-sectional area (CSA) of normal, control, and GDF11 mice at day 13. Data are mean \pm SD.

(F) Histogram of quadriceps CSA for normal, control, and GDF11 mice at day 13.

(G) Laminin staining (10x magnification, $100 \mu \mathrm{m}$ scale bar) of left hind limb quadriceps muscle cross-sections in normal, control, and GDF11 mice at day 13. Numerical data of mean myocyte CSA (data are mean \pm SEM) and number of fibers measured are shown in table below for $\mathrm{N}=4,4$, and 5 mice for normal, control and GDF11 mice respectively.

(H) Gene expression analyses of quadriceps muscle in normal (left of dashed vertical line, measured at day 13), control, and GDF11 mice at days 10 and 13. Data are represented as mean fold change \pm SD. All fold changes calculated using reference gene 18S.

Statistically significant differences are indicated at $\mathrm{p}<0.05\left(^{*}\right), \mathrm{p}<0.01(* *), \mathrm{p}<0.001$ $(* * *)$, and $\mathrm{p}<0.0001(* * * *)$. 
A
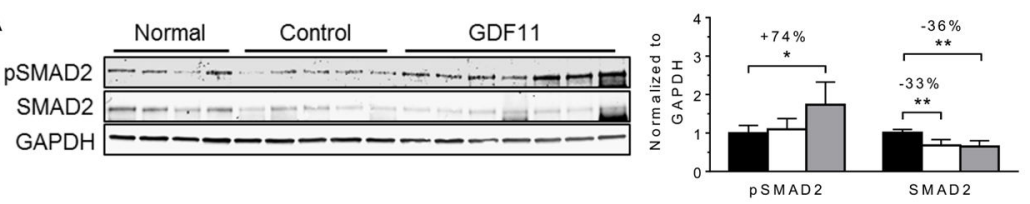

Normal

$\square$ control

GAPDH

-ーーー-ー-ー - - - -

PSMAD

$\square$ GDF11

B

Normal Control GDF11
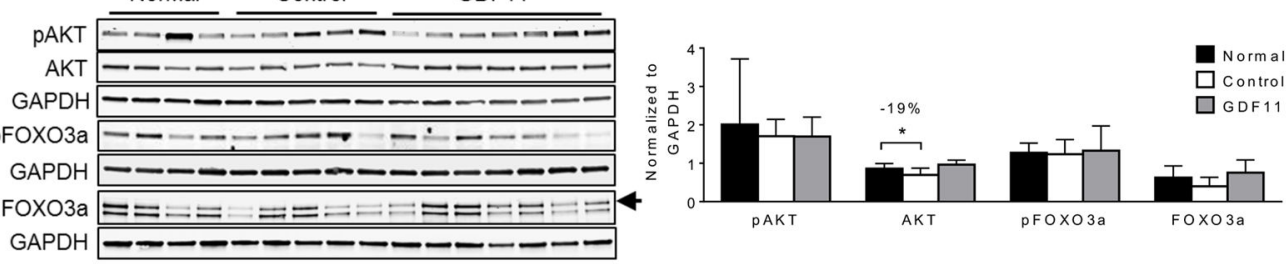

C
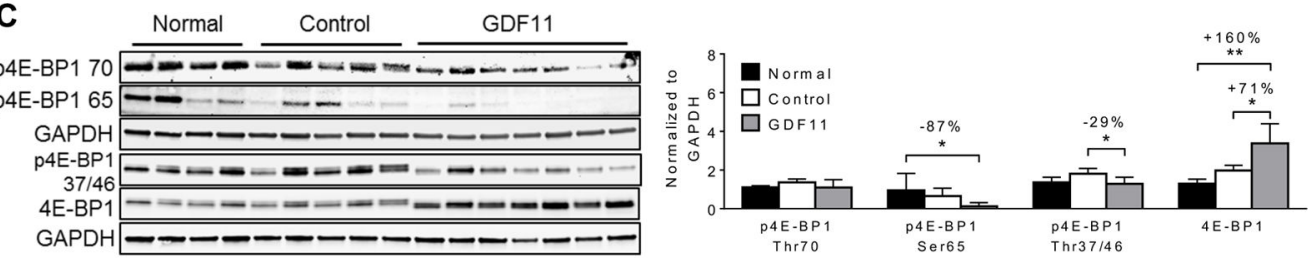

D
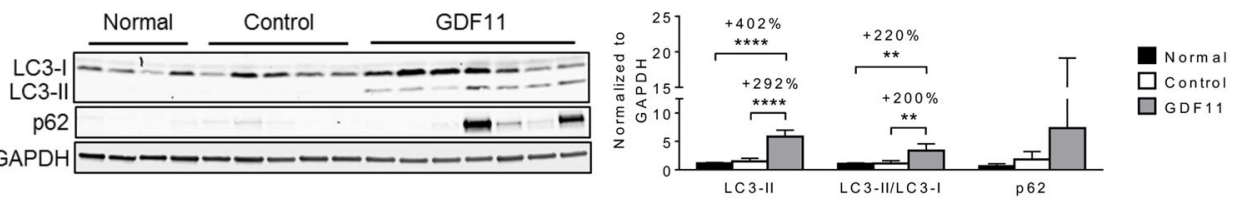

E
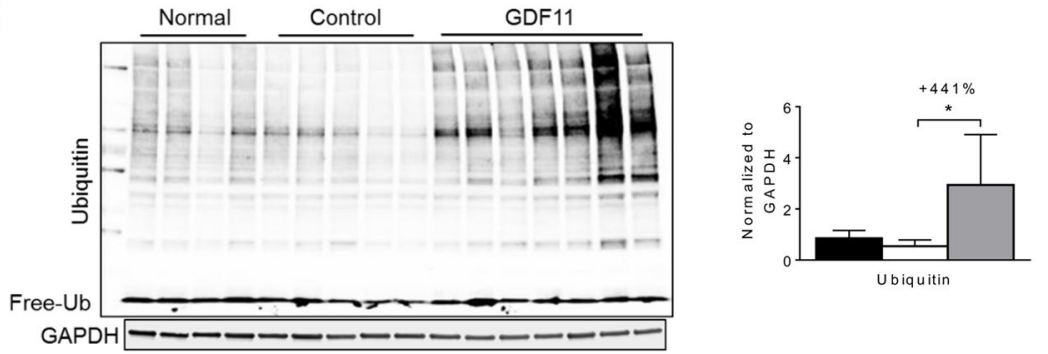

Normal

Control

Figure 4. Protein Regulatory Pathways in Quadriceps of Mice with GDF11 Overexpression Demonstrate Increased Degradation and Decreased Synthesis

(A-E) Western blotting analyses to determine activation of (A) SMAD2 signaling, (B) hypertrophy/atrophy pathways (arrowhead points to FOXO3a), (C) protein translation and synthesis, (D) autophagy, and (E) ubiquitination in normal, control, and GDF11 mice at day 13. Blots are presented on the left and protein levels quantified in graphs on the right. Horizontal lines with error bars indicate mean \pm SD.

Statistically significant differences are indicated at $\mathrm{p}<0.05(*), \mathrm{p}<0.01(* *)$, and $\mathrm{p}<$ $0.0001(* * * *)$. 